\title{
Ground stations for aeronautical and space laser communications at German Aerospace Center
}

\author{
Florian Moll*, Amita Shrestha, Christian Fuchs \\ German Aerospace Center (DLR), Institute of Communications and Navigation, 82234 Wessling
}

\begin{abstract}
Free-space laser communications are subject of current research and development in many research and industrial bodies. Long distance air-ground and space-ground can be implemented in future communication networks as feeder, backbone and backhaul links for various air- and space-based scenarios. The Institute of Communications and Navigation of the German Aerospace Center (DLR) operates two ground stations to investigate the communication channel and system: the Optical Ground Station Oberpfaffenhofen and the Transportable Optical Ground Station. The first one is a fixed installation and operated as experimental station with focus on channel measurements and tests of new developments. Various measurement devices, communication receivers and optical setups may easily be installed for different objectives. The facility is described with its dome installation, telescope setup and infrastructure. Past and current deployment in several projects is described and selected measurement achievements presented. The second ground station is developed for semi-operational use and demonstration purposes. Based on experience with the experimental ground station, this one is developed with higher level of integration and system robustness. The focus application is the space-ground and air-ground downlink of payload data from Earth observation missions. Therefore, it is also designed to be easily transportable for worldwide deployment. The system is explained and main components are discussed. The characteristics of both ground stations are presented and discussed. Further advancements in the equipment and capability are also presented.
\end{abstract}

Keywords: Free-space optical communications, optical ground station, transportable optical ground station, laboratory, facility, measurement system

\section{INTRODUCTION}

Aeronautical and space laser communications are gathering increasing interest for high-rate air-ground-, air-air-, airspace-, space-space- and space-ground-connections. Air-ground- and space-ground-connections require an Optical Ground Station (OGS) as ground node. Depending upon the specific experiment scenario, the OGS can be static or transportable. DLR has been developing two ground stations for its research activities: a fixed one (Optical Ground Station Oberpfaffenhofen, OGS-OP) for purely experimental activities and a Transportable Optical Ground Station (TOGS) for experiments, demonstrations and semi-operational use [1]. These are subject of this paper and are described in detail in the latter sections.

Setups with primary and secondary mirror in Cassegrain configuration are typical for medium sized and large telescope systems. This allows using the Cassegrain focus for the installation of experiments and instruments. However, a number of stations implement a Coudé-focus. This enables the stable installation of large and sophisticated experiments in a controlled environment. Typical telescope diameters range from $20 \mathrm{~cm}$ to $1.5 \mathrm{~m}$, which is small compared to the trends in Astronomy, however, more than suitable for free-space optical communication applications with limited ranges. Apertures of about 40 to $60 \mathrm{~cm}$ are typically sufficient for data reception from aircraft (with link distances up to $\sim 200 \mathrm{~km}$ ), but also for downlinks from low Earth orbit (up to 2’000 km link distance at low elevation angles) as well as links to geostationary satellites (up to $40^{\prime} 000 \mathrm{~km}$ distance). Deep-Space links, e.g. to the Moon and beyond, require telescope diameters of $1 \mathrm{~m}$ and more due to the large propagation distances. Depending on the chosen modulation format and detection technology, the use of Adaptive Optics (AO) may become a requirement, increasing the complexity of the OGS design. These AO systems are often also installed in the so called Coudé rooms.

Currently existing ground stations have their main focus on experimental and demonstration applications. European Space Agency (ESA) operates an OGS which is located at the Observatorio del Teide in Izaña on the Canary Island of Tenerife [2]. It consists of a $1 \mathrm{~m}$ Cassegrain telescope and facilitates a Coudé focus. It has been used in a number of electronic copy may be made for personal use only. Systematic or multiple reproduction, distribution to multiple locations via electronic or other means, duplication of any material in this paper for a fee or for commercial purposes, or modification of the content of the paper are prohibited. 
experiments, as e.g. for links to ESA's ARTEMIS satellite [3]. A ground station in Ukraine, the Main Astronomical Telescope (MAO) with an installed laser communication system, used the same satellite for experiments [4]. ESA's OGS was also deployed in experiments with NASA's Lunar Laser Communications Demonstration (LLCD) experiment [5]. Besides ESA's OGS, of course, NASA ground stations were used for LLCD as well [6]. These include the MITdeveloped LLGT (Lunar Lasercom Ground Terminal) [7], with four $40 \mathrm{~cm}$ receiver apertures located in White Sands, New Mexico, USA, and NASA-JPL's OCTL (Optical Communications Telescope Laboratory) [8], using a $1 \mathrm{~m}$ telescope at JPL's table mountain facilities, California, USA. The National Institute of Information and Communications Technology (NICT) operates a 1.5 m OGS in Tokyo, Japan. It has been used for satellite-to-ground experiments with the OICETS (Optical Inter-Orbit Communications Engineering Test Satellite) satellite [9]. A transportable ground station is under development in the framework of NICT's INNOVA (In-orbit and Networked Optical Ground Stations Experimental Verification Advanced Testbed) system as a testbed to verify new in-orbit terminals and space-ground communications [10].

\section{TRANSPORTABLE OPTICAL GROUND STATION}

\section{System overview}

The TOGS deployments are for experiment campaigns as well as for technology demonstrations and semi-operational use. Therefore, the system matches a quite high degree of integration. An image of the TOGS is shown in Figure 2.1. It shows the transport van, the unfolded and half folded TOGS and the operations room inside the van (from left to right). The actual receiver and transmitter system is positioned on four adjustable legs. The telescope is steered with a highprecision fork mount which is mounted on a foldable mast. Height of the optical axis is about $3 \mathrm{~m}$ when unfolded. The telescope and mount are pneumatically decoupled from the transport and storage compartment. The four legs can be detached and the telescope automatically folded into the compartment. This way, it can be conveniently transported in a van or an aircraft cargo. The TOGS is autonomous and includes all necessary infrastructure including power generator and uninterruptable power supply. The fork mount is constructed with carbon fiber and therefore very light-weight ( 500 $\mathrm{kg}$ ) and stiff. The TOGS van additionally hosts an operations room with several control computers, measurement devices for signal analysis and local area network (LAN).

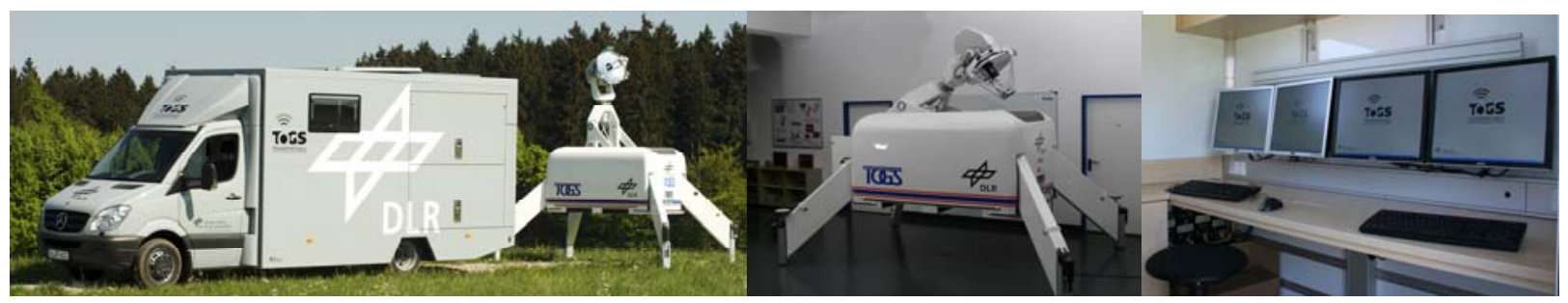

Figure 2.1. TOGS with its transport van (left), TOGS in folding process (middle), and operation room inside the van (right). Source: DLR.

\section{Hardware setup}

The main component of the TOGS is the $60 \mathrm{~cm}$ Ritchey-Chrétien type telescope with control and receiver electronics integrated in a backplane box (see Figure 2.1, middle image). A telescope close-up is shown in Figure 2.2 (left). The Ritchey-Chrétien design allows the short structure of the telescope. The telescope mirrors are milled from aluminum with optical quality close to glass mirrors and yet lighter, robust and easy to mount [11]. Hollow shaft precision torque engines are used for telescope steering. Absolute and incremental encoders are used on azimuth and elevation axes to achieve sub- $\mu$ rad positioning accuracy of the engines.

A block diagram of the main functional blocks is shown in Figure 2.2 (right). Two different control computers are used for antenna tracking, namely the PAT (Pointing, Acquisition and Tracking) Computer and Visual Tracker. The PAT computer controls the coarse tracking functionality and commands the mount engines controllers via CAN bus. It also interfaces to the attitude sensor via RS-232 which helps in the initial alignment of the TOGS. The Visual Tracker is integrated in the backplane of the telescope and connected to the Narrow Field Of View (NFOV) camera and the Wide Field Of View (WFOV) camera. NFOV camera is a near-infrared camera used as a closed-loop tracking sensor and is placed in the optical system behind the telescope (Figure 2.3). The tracking signals from the NFOV camera are sent to the PAT computer via network. The WFOV camera is mounted and co-aligned at the side of the telescope and is used for 
visual feedback. A Dual-Antenna GPS system is installed at two ends of the TOGS platform which provides precise positioning and heading information. All computers and GPS receivers are connected to the LAN for internal communication. The optical and electrical signals are transmitted from the telescope backplane box to the operations room and PAT Computer by means of a slip ring integrated in the mount azimuth axis.
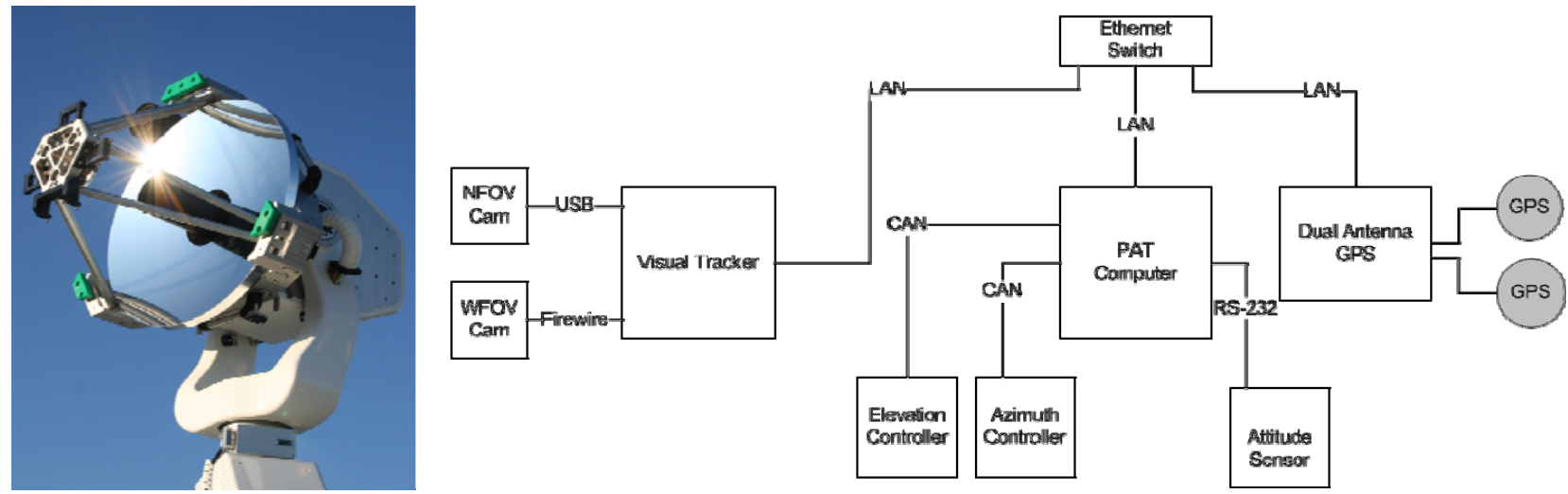

Figure 2.2. Left: $60 \mathrm{~cm}$ Ritchey-Chrétien telescope of TOGS (source: DLR). Right: Hardware functional block diagram. NFOV: Near Field of View Camera; WFOV: Wide Field of View Camera.

The block diagram of the optical system in the telescope backplane box is shown in Figure 2.3. The beam received from the transmitter is split to the tracking camera path and Receiver Front End (RFE) path. The latter is used for data reception. In order to keep complexity low, no additional fine pointing system is installed. Therefore, the pointing accuracy solely depends on the closed-loop tracking using the NFOV camera, the controllers and engines. A flippable and static optical filter protect from background light. The collimation lens position is adjustable along the optical axis to collimate the beam for varying object distances. In order to support optical acquisition and tracking on the counter terminal (in air or space), a beacon laser system is installed at the side of the telescope mount. A bi-static transmitter telescope setup is here for transmitter diversity (not seen in the block diagram).

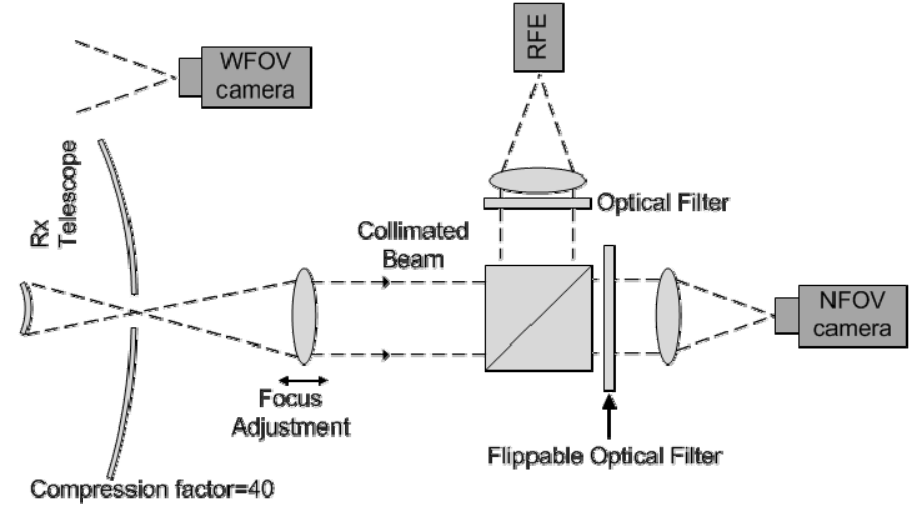

Figure 2.3. Block diagram of optical system integrated in backplane box behind telescope.

RFE: Receiver Front End; WFOV: Wide Field of View; NFOV: Narrow Field of View.

\section{Software setup}

The control software in the PAT computer is the brain of the whole system. The software has been developed in a Linux (Debian) environment using $\mathrm{C}++$ language because for its inter-operability in different operating systems, flexibility and real-time abilities. The software structure comprises the user interface layer including graphical user interface, the device layer including drivers for devices (like motor controllers, sensors, joystick, GPS), and the system layer includes the core PAT system. The user interface layer communicates to other layers via network thereby enabling the remote control ability of the terminal [11]. 
The PAT system of the TOGS comprises pointing, acquisition and tracking modes. The PAT process works as follows: Initially, terminals are set in a line-of-sight open-loop pointing mode to find each other by following the partner's initial position. The PAT software receives the instantaneous position of the counter terminal (based on orbit or GPS information) via network and can keep tracking its counter terminal in open-loop. This open-loop tracking is precise enough so that the counter terminal hits the beacon signal coming from the TOGS and starts its closed-loop optical tracking. Once the counter terminal has acquired the signal from the TOGS, the optical signal from the counter terminal will be visible as a spot in the tracking camera behind the telescope. The visual tracking software reads the image from the tracking camera and calculates position error (difference between current position of the spot and the target position) and provides this information to the tracking manager, the component of the software that collects all necessary information, analyses and commands the mount to move precisely to keep the tracking lock. The tracking manager then commands the mount which moves accordingly. In this way, the PAT process continues thus keeping link lock between the terminals. The control of the telescope mount by the software is shown in Figure 2.4. The Pointing Angle Generator creates Azimuth (Az) and Elevation (El) vectors based on initial configuration and the counter terminals initial position. Corrected vectors are created by the Pointing model (Az', El'). These are used by the Tracking Manager, together with the real-time signals form the Tracking Camera to control position and velocity of the mount engines.

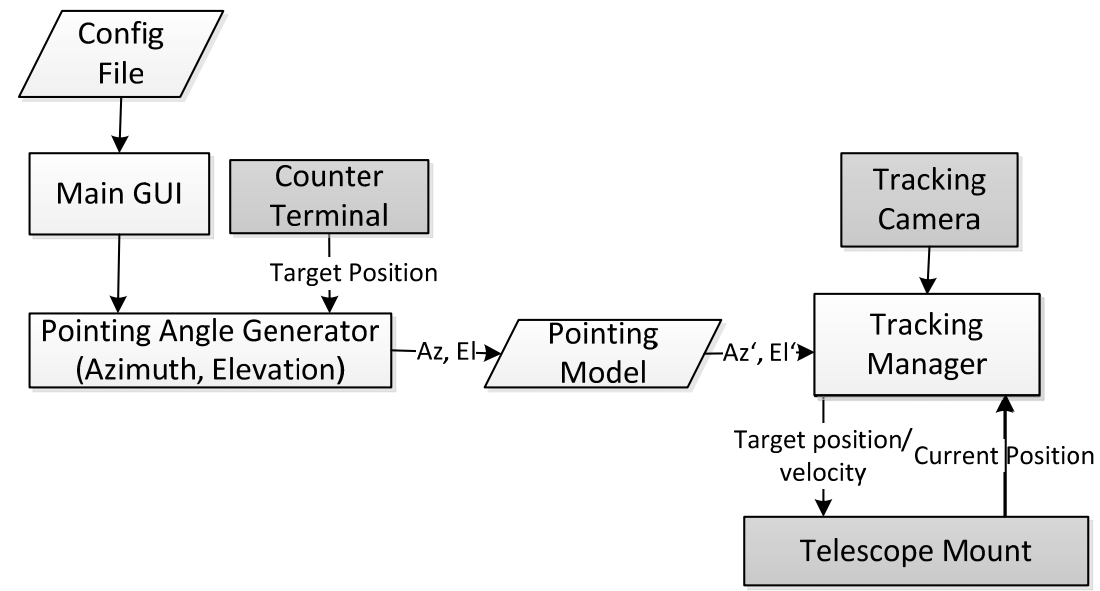

Figure 2.4. Block diagram of the TOGS control software. The bright blocks indicate software modules, the dark blocks indicate interfaces with hardware.

\section{Calibration}

TOGS consists of non-ideal mechanical structures and optical systems resulting in errors in the alignment. Additionally, the ground platform on which the TOGS would be placed is usually not perfectly flat. Such imperfections in the mechanical and optical system alignment cause errors in the PAT process. These errors can be offsets in the azimuth and elevation, non-perpendicularity of azimuth and elevation axes, non-perfect alignment between the optical axes, tilt of azimuth axis towards North or East, telescope flexure etc. [11]. A set of these errors are named as pointing model. It is a well-known problem in telescope control including astronomical devices. Similar to astronomical telescopes, such errors are modelled and compensated by star calibration methods [12]. Stars are used as reference targets as they are widely distributed in the sky and positions are very accurately known. For star calibration, first the TOGS points to a star that is visible on the tracking camera. The error offset of the position of the star is seen on the camera and this error is compensated by providing manual offsets. The original position of the star and the compensated error values are stored. The process is repeated for more than 10 stars well-distributed in the sky for different azimuth and elevation angles. All the measurements are then analyzed and fitted to calculate the pointing model of the TOGS. After the calibration, the set of model parameters is loaded during the subsequent system starts.

\section{Former, current and future deployment}

The TOGS has been successfully deployed in various experiments with counter terminals on ground, aircraft and satellite. In 2011 the first downlink from DLR aircraft (Dornier 227) was successfully conducted with the TOGS in the framework of the DLR internal project VABENE [1][13]. Data rates up to $1.25 \mathrm{Gbit} / \mathrm{s}$ and link distances up to $90 \mathrm{~km}$ could be achieved [13]. In 2013, more challenging (with non-ideal weather condition, large vibration and very high 
speed of moving aircraft) data downlink experiment was successfully accomplished. In this experiment, the data from an optical terminal installed in fast flying jet aircraft (Tornado, with a speed up to 0.7 Mach) was received within the framework of DODFAST project [14]. Even in such harsh conditions, TOGS was able to achieve tracking lock for distances up to $79 \mathrm{~km}$ and data communication up to $50 \mathrm{~km}$. The tracking error during the experiment was well below $100 \mu \mathrm{rad}$ as shown in Figure 2.5 that shows the excerpt of the tracking error during one of the experiments runs.

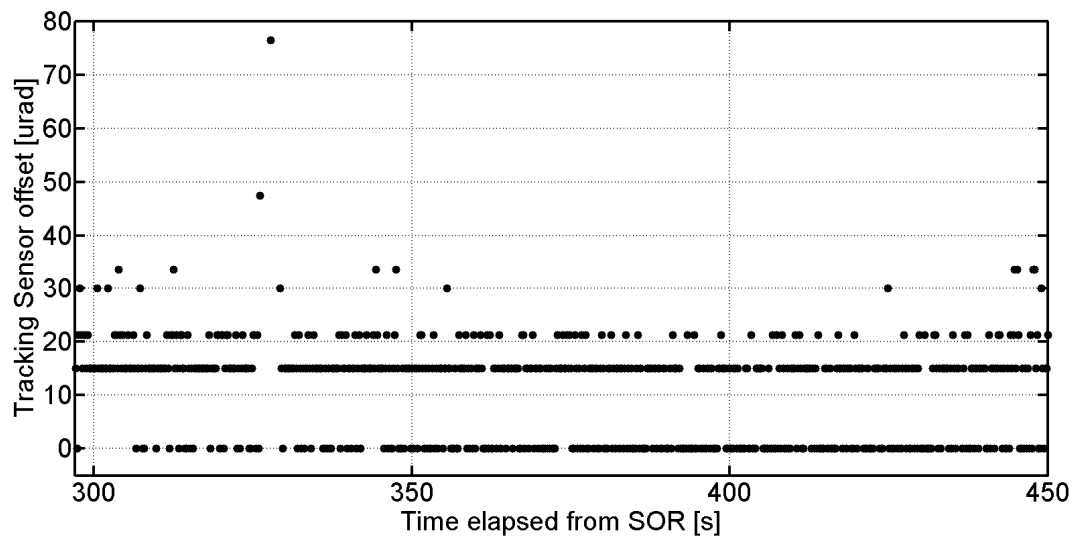

Figure 2.5. Excerpt of tracking error measured by TOGS during DODFAST flight \#02, run \#12 w.r.t time elapsed from start of run (SOR).

Satellite tracking ability of the TOGS has also been successfully tested with sunlit ISS with NASA's OPALS system as test source [15]. In this project, successful closed-loop tracking with sun-lit ISS was tested. Currently, the further improvement of the blind pointing accuracy of the TOGS is on-going for foreseen optical satellite downlinks in the OSIRIS project [16]. Recently in June, an inter-island link of $142 \mathrm{~km}$ between La-Palma and Tenerife was successfully tested with a counter terminal in Tenerife and TOGS in La-Palma within the project HiCLASS-ROS (Highly Compact Laser Communication Terminals for Robotic Operations Support). Because of the good performance and high-quality of the TOGS software, it is planned to be ported to the further development of the fixed ground station.

\section{OPTICAL GROUND STATION OBERPFAFFENHOFEN}

\section{System overview}

The OGS-OP serves as the experimental station for free-space optical communications research at DLR. In contrast to the TOGS, which has a rather high degree of integration, the OGS-OP is very flexible regarding installation of measurement devices and changes in the optical setup. It can therefore be seen as the ground station laboratory. The system is set up in a way that makes it easy to install and change a variety of custom made and off-the-shelf components. A particular core setup defines the basic system which is usually not changed amongst different projects. This core system comprises the central telescope and the collimation optics on the backplane, the fine pointing assembly and the tracking sensors. It is located at the DLR site Oberpfaffenhofen near Munich, Germany. The main specifications are listed in Table 1.

Table 1. Main specifications of Optical Ground Station Oberpfaffenhofen.

\begin{tabular}{|l|l|l|}
\hline \multicolumn{1}{|c|}{ Item } & \multicolumn{1}{|c|}{ Value } & \multicolumn{1}{c|}{ Unit } \\
\hline Geographic latitude & $48.0848^{\circ}$ & North \\
\hline Geographic longitude & $11.2780^{\circ}$ & East \\
\hline Altitude above sea level & 602 & $\mathrm{~m}$ \\
\hline Telescope primary mirror diameter & 40 & $\mathrm{~cm}$ \\
\hline Focal length (Cassegrain) & 3.6 & $\mathrm{~m}$ \\
\hline
\end{tabular}


The infrastructure of the OGS-OP comprises the building facilities, several control computers for service, measurement and PAT, a laboratory LAN, a time synchronization system, a switching matrix for the control computers, a weather station, a test link, optional UHF and RF links, a storage raid and webcams for monitoring. An image of the ground station dome on top of the institutes building and the hosted telescope is given in Figure 3.1. The building facilities comprise the housing of the telescope (clamshell dome), an operations room beneath and an operations container on the roof platform. The telescope base is a cemented shaft built upon the ceiling of the operations room. The ceiling is designed quite strong (35 cm thickness) to obtain high stiffness. The dome is placed on a concrete ring that lies on a special damping layer to decouple the dome with its inner floor from the telescope base.
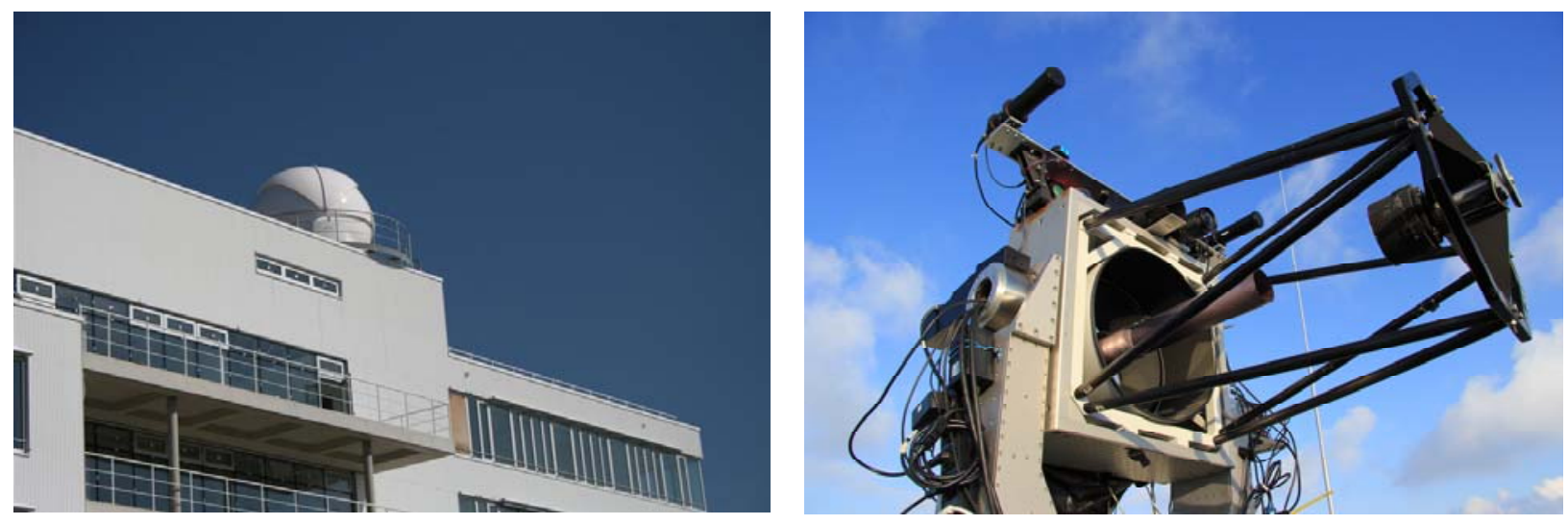

Figure 3.1. Image of ground station dome on top of the institutes building (left) and Cassegrain telescope (right). Source: DLR.

\section{Hardware and software setup}

The functional block diagram of the basic setup is given in Figure 3.2. Central parts are the OGS Service, the PAT Control, the FPA Control and multiple Measurement DAQs with corresponding sensors. The latter ones are, depending on the actual measurement, a power meter, a pupil camera, a wave-front sensor, a focus camera, a differential image motion monitor and a receiver front end with bit error rate tester or data sampling device. The PAT Control interfaces to a NIR and VIS camera (WFOV Cam) to facilitate the optical closed loop control of the mount motors. The FPA Control interfaces to the NFOV camera for control of the fast steering mirror (Mirror drive). The computers are networked via an Ethernet switch for data transfer to the raid system, data exchange during runtime of the measurements and to connect the monitoring webcams. The time synchronization is realized with a GPS receiver card in the service computer (OGS Service). The time signal is distributed to the PAT Control, FPA Control and Measurement DAQs via IRIG timecodes. A KVM (Keyboard Video Mouse) matrix is connected to all computers to enable flexible device operation. A DVI (Digital Visual Interface) recorder is further connected to record screen images for documentation. The weather station comprises a thermometer, hygrometer, barometer and anemometer. The UHF (Ultra High Frequency) and an RF (Radio Frequency) links are available on site to support TMTC (TeleMetry and TeleCommand) needs of particular project, most often for experiments involving aircraft. The test link is located in a distance of about $700 \mathrm{~m}$ on top of a neighboring office building. An outdoor box hosts two laser sources in C-band, one at $850 \mathrm{~nm}$ and all other optical and electronic devices. Air interface is a standard double glass window. The testlink is remotely operated via an electronic power supply. A remote computer is used to operate a function generator for laser modulation and an oscilloscope. Furthermore, a Laser Ethernet Transceiver (LET) is installed to transmit test videos from a webcam. The optical signals and LET signals are used for test and calibration of the measurement devices in the OGS-OP in advance of the actual experiments and to measure communication performance of particular setup within this test range.

The control software of the OGS-OP is very similar to the TOGS one and can be seen as a precursor. The PAT process is similar to the one described in section 2. However, the different building blocks do not share a common programming language. They are therefore separated in different modules implemented in $\mathrm{C}^{++}$, Matlab and LabView. All modules are interfaced for full functionality. 


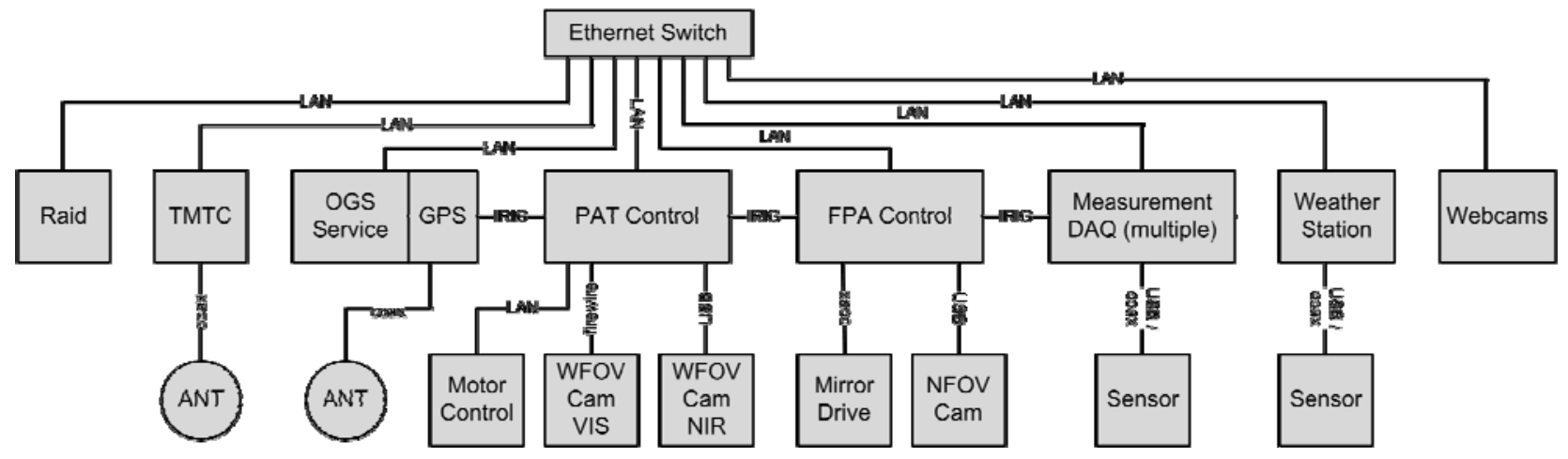

Figure 3.2. Functional block diagram of OGS-OP hardware. The coax lines denote arbitrary electrical signals for measurement and control. TMTC: TeleMetry TeleCommand; PAT: Pointing, Acquisition and Tracking; FPA: Fine Pointing Assembly; DAQ: Data AcQuisition; WFOV: Wide Field of View; NFOV: Near Field of View.

\section{Optical measurement setup}

Core element of the measurement setup is the Cassegrain telescope with $40 \mathrm{~cm}$ primary mirror diameter. The telescope is hold by a custom made aluminum fork mount. Azimuth and elevation axes are steered by two gearbox servo drives. The block diagram of the measurement setup is shown in Figure 3.3 (left). The black bar indicates all subsystems that are held by the telescope mount, i.e. which all are moved together: the big measurement telescope (Cassegrain), the camera telescope with WFOV camera, the beacon optics and the additional smaller measurement telescopes (mostly refractors). The mount is controlled by using signals from a co-aligned WFOV tracking camera. Collimation optics behind the telescope compress the captured beam by factor of 40 and place a pupil image on the fine pointing assembly (FPA). An optical coupling system distributes the beam to the RFE and the measurement instruments. The NFOV camera creates inputs to the control of the FPA.
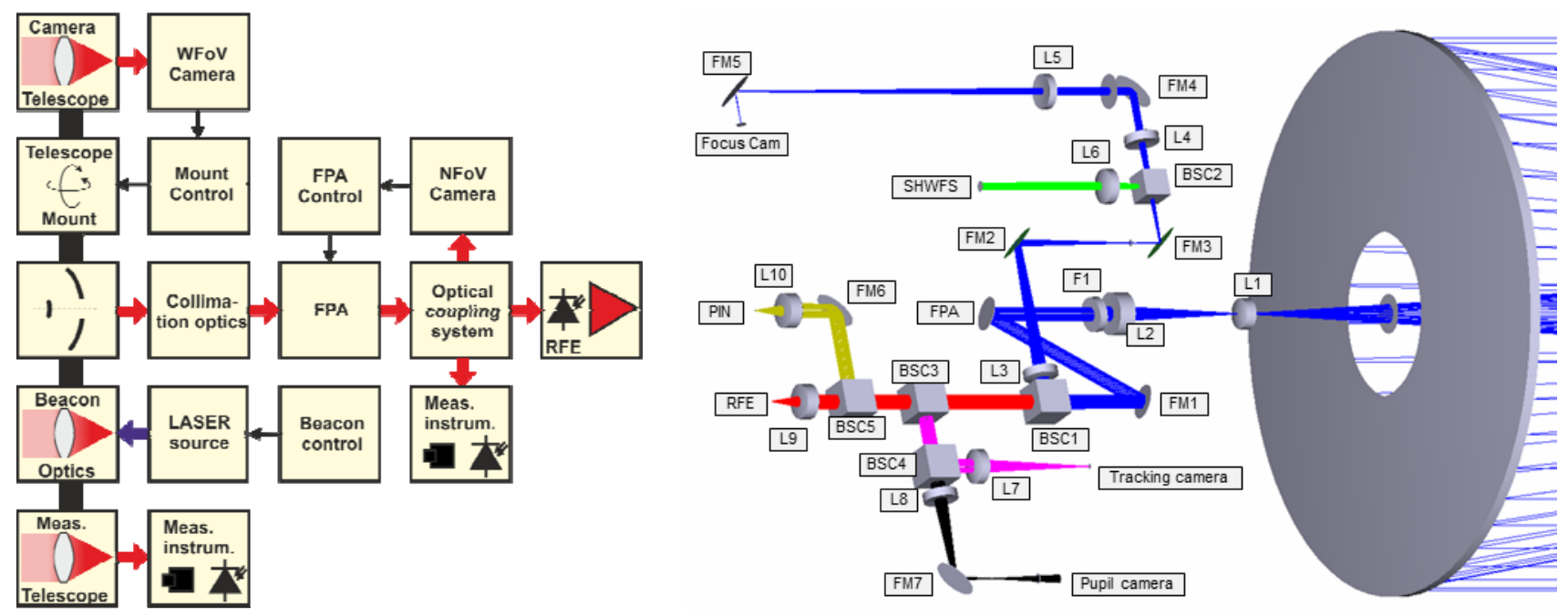

Figure 3.3. Basic block diagram of OGS-OP optical system (left). The black bar indicates mechanical connection of the functional blocks. The right graphs depicts the ray-trace of the optical system behind the telescope of the setup for the A/C-measurement campaign in the ALTAO project. WFoV: Wide Field of View; NFoV: Narrow Field of View; FPA: Fine Pointing Assembly; RFE: Receiver Front End; SHWFS: Shack-Hartmann Wave-Front Sensor; LX: Lens X; FMX: Folding Mirror X; BSCX: Beam Splitter Cube X; FX: Filter X.

The optical setup is placed on an aluminum bench which is flanged to the back of the telescope. Depending on the individual measurement needs, the assembly differs for different projects. The measurement setup of the ALTAO measurement campaign (aircraft-ground link) [17] is shown in the right graph of Figure 3.3. The received beam is 
collimated and a pupil is imaged onto the FPA (active mirror) under use of lenses L1 and L2. The optical bandpass filter F1 is the first line of defense against background light. Folding mirrors (FM1-FM7) are used to fit the system onto the optical bench. Beam splitter cubes are used to distribute beams of different power to the individual measurements instruments. BSC1 splits the incoming beam towards the lower and upper part of the optical bench. The upper part hosts the Shack-Hartmann Wave-Front Sensor (SHWFS) and the Focus camera. The beam in the upper part is split by BSC2. The lens system L3/L6 creates a pupil image at the lenslet array of the SHWFS. The lenses L3/L4/L5 create a long effective focal length for the Focus Cam. In the lower part of the optical bench, BSC3/4/5 distribute the beam to the Tracking camera, the Pupil camera, the RFE and the PIN (power meter). Lens L8 creates a pupil image on the Pupil camera, L7/9/10 act as focusing lenses.

\section{Former, current and future deployment}

The OGS-OP was part of several projects for test of communication terminals and measurements of the propagation channel in satellite and aircraft scenarios. LEO satellite downlink (LEO-DL) measurements were undertaken in 2006 and 2009 with the Japanese OICETS satellite $[18,19]$. Aircraft-ground data downlinks (A/C-DL) experiments were done in the framework of the project ARGOS and VABENE [20,21]. Channel measurements in the same scenario were done in the ALTAO project to characterize the optical turbulence for adaptive optics specification [17]. Feasibility of Quantum Key Distribution (QKD) in the space-ground scenario was demonstrated by an air-ground test campaign with a QKD transmitter installed in an aircraft and a QKD receiver integrated in the OGS-OP [22]. Tracking performance was analyzed for several deployments. Figure 3.4 shows excerpts of the RMS (Root Mean Squared) radial tracking error during a LEO-ground link (left) and an aircraft-ground link (right) over a time span of 150 s, respectively. Both measurements were taken in nighttime conditions. The LEO-DL measurement was taken during the KIODO campaign on 28.08.2009 at around $4 \mathrm{am}$ local time. The A/C-DL measurement was taken during the QKD-campaign on 09.03.2011 at around 7 pm local time. Both measurements show the RMS tracking error to be at around $15 \mu$ rad, with a few outliers. The tracking systems were partly different in both cases. In the LEO-DL experiment, no fine pointing assembly was used. Here, the NFOV camera was installed in the backplane of the telescope and used for fine control of the mount once the signal was in the field of view. In the A/C-DL case, a fine pointing assembly was installed as shown in the setup in Figure 3.3 (right). Reason for implementation of the FPA is the unknown trajectory of the transmitter terminal. Since orbit data are deterministic (to some accuracy), the trajectory is known a priori and the mount movement can be controlled accordingly smooth. In the A/C-DL case, the trajectory is unknown, the aircraft position is sent via telemetry to the ground station. This causes the need of the optical tracking to be faster, i.e. installation of the FPA was necessary. Eventually, the error in the case of the A/C-DL is slightly lower than in the case of the LEO-DL and shows more steady behaviour.
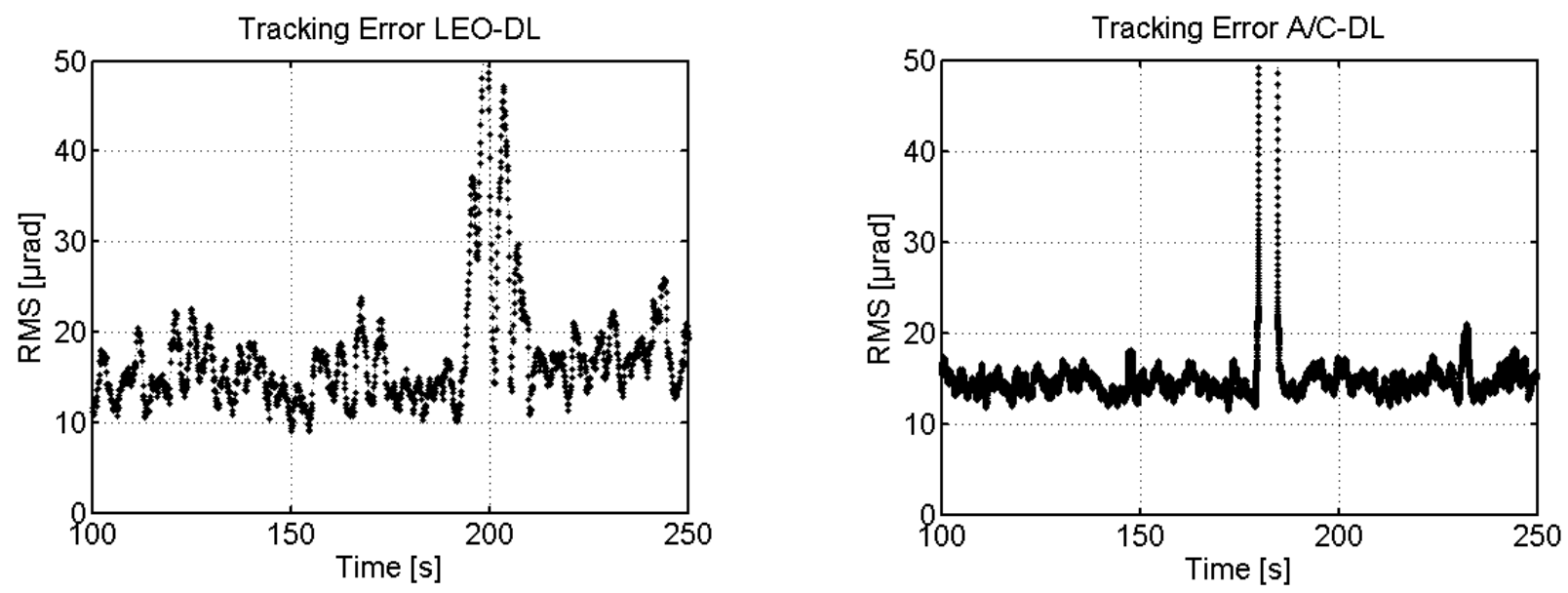

Figure 3.4. Excerpts of tracking performance for a LEO downlink (left) and A/C downlink (right) in nighttime conditions. The LEO measurements were taken during the KIODO campaign in 2009, the A/C measurements during the QKD-campaign in 2012.

Channel measurements in LEO-DL were also achieved [19][23]. Intensity and wave characteristics were measured in nighttime conditions depending on link elevation. Values of Fried parameter between $1 \mathrm{~cm}$ and $11 \mathrm{~cm}$ with increasing 
link elevation occurred. Intensity scintillation index was observed up to values of 2.6, power scintillation index values were observed up to values of 0.4 (40 $\mathrm{cm}$ aperture). Correlation time of the power scintillation was seen to vary between $0.5 \mathrm{~ms}$ and $2 \mathrm{~ms}$. Also fading characteristics, temporal behavior of the channel and wave-front measurement were analyzed and presented and are further discussed in [23-25]. Further measurement campaigns are currently planned with NASA's OPALS terminal [15]. Furthermore, future deployment is planned for the evaluation and measurement campaign with DLR's laser transmitters in LEO within the OSIRIS project [26].

\section{Current developments}

Future measurement setups will need a more laboratory like clean and calm environment as the OGS-OP can offer it to date. Therefore, the setup of a Coudé- and Nasmyth system in planned. A future Coudé-room is planned to be located in the operations room below the astronomical dome, illustrated in Figure 3.5 (left) with the basic beam path from the telescope to the room below. A more detailed view of the beam path along the telescope is shown in Figure 3.5 (right). A system of four folding mirrors guides the beam through the elevation axis, along the outer arm of the fork mount and through the azimuth axis. A hollow pillar and a hole in the ceiling give path to the inside of the Coudé-room. For multiple ways of deployment, a switching concept is planned to allow operation of the telescope with Cassegrain, Nasmyth and Coudé focus. This will enable different optical setups using any of the three foci. A controllable optical element before the Cassegrain focus will be used to switch between the three different modes. The concept of this mode switching is illustrated in Figure 3.5 (right). A right angle mirror is set on a translation stage to move to three different positions. Positions \#1 corresponds to Coudé mode, position \#2 to Nasmyth mode and position \#3 to Cassegrain mode.

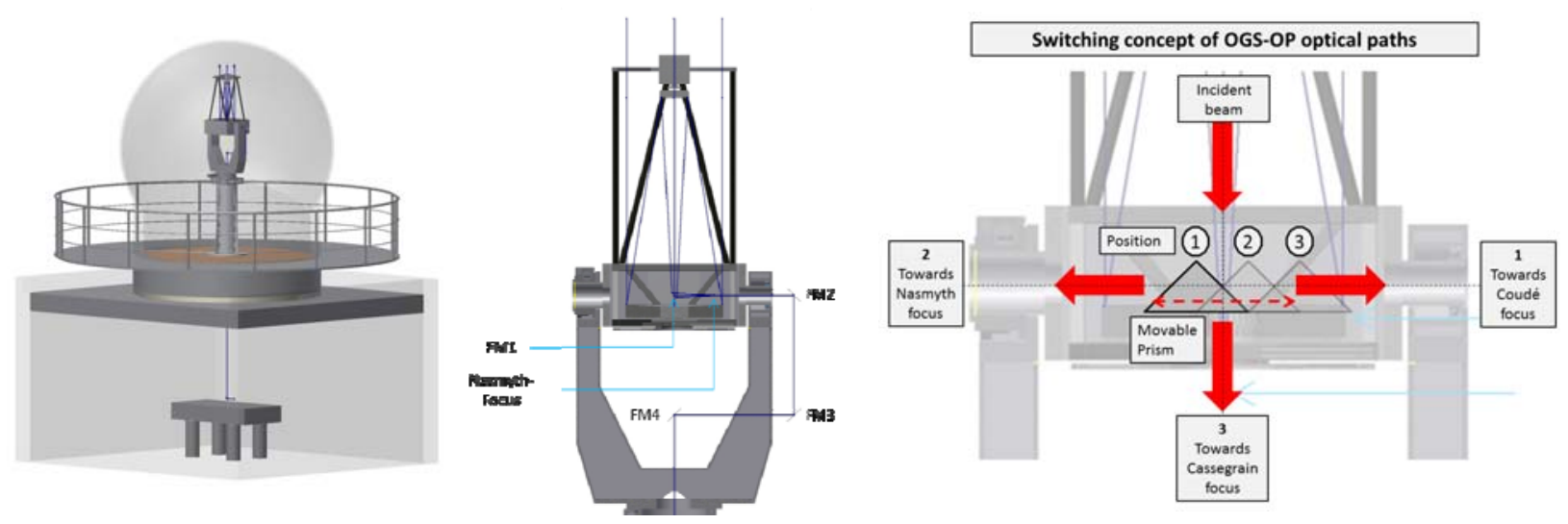

Figure 3.5. CAD of Coudé room (left), optical path along the telescope mount (middle) and switching concept (right).

Furthermore, the TOGS PAT software will be ported to OGS-OP. This way, better tracking and blind pointing accuracies are expected. New engines integrated in a stiffer mount structure will also contribute to higher blind pointing accuracy, thus enabling the deployment of the OGS-OP also in experiment with geostationary satellites.

\section{SUMMARY}

Two ground stations for experiments in free-space optical communications for aeronautical and space scenarios were presented. The Transportable Optical Ground Station is very flexible in terms of deployment at different locations. Therefore, demonstration and measurements can be undertaken at nearly any place in the word. The Optical Ground Station Oberpfaffenhofen is more flexible in terms of setup installation. Since the optical bench behind the telescope is similar to a laboratory table, measurement setups can be tested and changed very easily. Performance of the ground stations in terms of tracking error was shown. Former and future deployment of the ground station is explained and current developments outlined. A Coudé system is in plan for the OGS-OP as well as a mayor refurbishment of the mechanical structure and new motors for higher precision. Furthermore, TOGS software will be ported to OGS-OP. The software is being improved for better tracking and blind pointing accuracy. 


\section{ACKNOWLEDGEMENTS}

The authors would like to thank all members of the institute's research groups Advanced Optical Technologies and Optical Communication Systems who were involved in the design, assembly and operation of the TOGS and OGS-OP. Special thanks goes to Mr. Martin Brechtelsbauer as lead engineer in the TOGS project and Dr. Markus Knapek who was responsible for the initial setup of the OGS-OP.

\section{REFERENCES}

[1] C. Fuchs, M. Brechtelsbauer, J. Horwath, A. Shrestha, F. Moll, D. Giggenbach, and C. Schmidt, "DLR's Transportable Optical Ground Station," Proc. of Applications of Lasers for Sensing and Free Space Communications (OSA Technical Digest), 2013.

[2] Z. Sodnik, B. Furch, and H. Lutz, “The ESA Optical Ground Station - Ten Years Since First Light,” ESA bulletin, vol. 132, 2007, pp. 34-40.

[3] A. Alonso, M. Reyes, and Z. Sodnik, "Performance of satellite-to-ground communications link between ARTEMIS and the Optical Ground Station,” Proc. SPIE 5572, Optics in Atmospheric Propagation and Adaptive Systems VII, 372 (November 11, 2004), 2004.

[4] V. Kuzkov, D. Volovyk, S. Kuzkov, V. Caramia, Z. Sodnik, and S. Pukha, "Laser Ground System for Communication Experiments with ARTEMIS,” Proc. International Conference on Space Optical Systems and Applications (ICSOS) 2012, 2012.

[5] M. Sans, Z. Sodnik, I. Zayer, and R. Daddato, "Design of the ESA Optical Ground Station for Participation in LLCD,” Proc. International Conference on Space Optical Systems and Applications (ICSOS) 2012, 2012.

[6] B.S. Robinson, D.M. Boroson, D.A. Burianek, and D.V. Murphy, "The lunar laser communications demonstration,” Proc. of International Conference on Space Optical Systems and Applications (ICSOS) 2011, 2011.

[7] D.V. Murphy, J.E. Kansky, M.E. Grein, R.T. Schulein, M.M. Willis, and R.E. Lafon, "LLCD operations using the Lunar Lasercom Ground Terminal," Proc. of SPIE Vol. 8971 (Free-Space Laser Communication and Atmospheric Propagation XXVI), 2014.

[8] A. Biswas, J.M. Kovalik, M.W. Wright, W.T. Roberts, M.K. Cheng, K.J. Quirk, M. Srinivasan, M.D. Shaw, and K.M. Birnbaum, "LLCD Operations Using the Optical Communications Telescope Laboratory (OCTL),” Proc. of SPIE Vol. 8971 (Free-Space Laser Communication and Atmospheric Propagation XXVI), 2014.

[9] M. Toyoshima, K. Takizawa, T. Kuri, W. Klaus, M. Toyoda, H. Kunimori, T. Jono, Y. Takayama, N. Kura, K. Ohinata, K. Arai, and K. Shiratama, “Ground-to-OICETS laser communication experiments,” Proc. of SPIE Vol. 6304 (Free-Space Laser Communications VI), 2006.

[10] T.Yamashita, M.Shimizu, S. Nishimura, T.Nishizawa, M.Nakai, Y. Munemasa, Y.Koyama, Y. Takayama, and M.Toyoshima, "In-orbit and Networked Optical Ground Stations Experimental Verification Advanced Testbed (INNOVA): The High-performance and Compact Ground-tracking System,” Proc. International Conference on Space Optical Systems and Applications (ICSOS), 2014.

[11] A. Shrestha and M. Brechtelsbauer, "Transportable optical Ground station for high-speed free-space laser communicaiton,” SPIE preceedings, 2012.

[12] A. Hempelmann, J.N. Gonzalez Perez, J.H.M.M. Schmitt, and H.J. Hagen, "The Hamburg Robotic Telescope: A Test Report,” Proceedings of 13th Cool Stars Workshop, 2004.

[13] F. Kurz, D. Rosenbaum, J. Leitloff, O. Meynberg, and P. Reinartz, "Real time camera system for disaster and traffice monitoring," International Conference on Sensors and Models inPhotogrammetry and Remote Sensing, 2011.

[14] F. Moll, W. Mitzkus, J. Horwath, A. Shrestha, M. Brechtelsbauer, L.M. Navajas, A.L. Souto, and D.D. Gonzalez, "Demonstration of high-rate laser communications from fast airborne platform: flight campaign and results," Proc. SPIE 9248, Unmanned/Unattended Sensors and Sensor Networks X, 92480R, 2014.

[15] B.V. Oaida, M.J. Abrahamson, R.J. Witoff, J.N.B. Martinez, and D.A. Zayas, “OPALS: an optical communications technology demonstration from the International Space Station,” IEEE, 2013, pp. 1-20.

[16] C. Fuchs, M. Brechtelsbauer, and D. Giggenbach, "Verification of Ground Station Diversity For Direct Optical TTC-Downlinks from LEO Satellites by means of an Experimental Laser-Source," 5th ESA International Workshop on Tracking, Telemetry and Command Systems for Space Applications, 2010. 
[17] K. Shortt, D. Giggenbach, R. Mata-Calvo, F. Moll, C. Fuchs, C. Schmidt, J. Horwath, J. Yeh, V. Selvaraj, and R. Banerjee, “Channel characterization for air-to-ground free-space optical communication links,” Proc. SPIE 8971 (Free-Space Laser Communication and Atmospheric Propagation XXVI), 2014.

[18] F. Moll, “KIODO 2009: Trials and analysis,” GOLCE 2010, 2010.

[19] N. Perlot, M. Knapek, D. Giggenbach, J. Horwath, M. Brechtelsbauer, Y. Takayama, and T. Jono, "Results of the optical downlink experiment KIODO from OICETS satellite to Optical Ground Station Oberpfaffenhofen (OGSOP)," Free-Space Laser Communication Technologies XIX and Atmospheric Propagation of Electromagnetic Waves, O. Korotkova and S. Mecherle, eds., 2007, p. 645704.

[20] J. Horwath and C. Fuchs, "Aircraft to ground unidirectional laser-communications terminal for high-resolution sensors,” Proc. SPIE, Free-Space Laser Communication Technologies XXI, 2009.

[21] C. Schmidt, J. Horwath, A. Shrestha, F. Moll, M. Brechtelsbauer, and C. Fuchs, "High-speed, high-volume optical communication for aircraft," SPIE Newsroom, 2013.

[22] S. Nauerth, F. Moll, M. Rau, C. Fuchs, J. Horwath, S. Frick, and H. Weinfurter, "Air-to-ground quantum communication,” Nature Photonics, vol. 7, 2013, pp. 382-386.

[23] F. Moll and M. Knapek, "Free-space laser communications for satellite downlinks: Measurements of the atmospheric channel," Proc. of 62nd International Astronautical Congress, 2011.

[24] M. Knapek, "Adaptive Optics for the Mitigation of Atmospheric Effects in Laser Satellite-To-Ground Communications,” Technische Universität München, 2010.

[25] F. Moll, "Experimental analysis of channel coherence time and fading behavior in the LEO-ground link,” Proc. of the International Conference on Space Optical Systems and Applications 2014, 2014.

[26] C. Schmidt, M. Brechtelsbauer, F. Rein, and C. Fuchs, "OSIRIS payload for DLR's BiROS satellite," International Conference on Space Optical Systems and Applications 2014, 2014. 